\title{
Improved Information Recovery in Phase Contrast EM for non-two-fold symmetric Boersch phase plate geometry
}

\author{
E. Majorovits and R. R. Schröder
}

Max-Planck-Institute for Medical Research, Jahnstr.29, D-69120 Heidelberg, Germany

Phase contrast imaging is a common mode for visualizing weak phase objects in LM. Recently this imaging mode has been realized in TEM using simple carbon film as a Zernike type phase plate [1]. Additionally, an electrostatic Boersch phase plate has been proposed [2,3]. With this new mode of TEM imaging it is possible to recover all spatial frequencies from only two complementary images, one bright field and one phase contrast image [1]. This is of special interest when studying beam sensitive samples [4]. In principle, the electrostatic Boersch phase plate should be superior to the carbon film phase plate, as there is no electron-amorphous film interaction limiting resolution. Instead it uses a phase-shifting electrostatic potential, which is applied within a circular electrode structure (figure 1). However, the geometry of the supporting rods of the ring electrodes affects the object information, which can be retrieved from the recorded images. It is shown here that the originally proposed centrosymmetric design can be optimized for an almost complete information recovery using non-two-fold geometries for the supporting rods. A further improvement of the reconstructed object can then be obtained by correcting structure factor amplitudes.

Bright field and phase contrast TEM images of different test objects were simulated assuming a 2-fold (figure 1a) or 3-fold (figure 1b) symmetric Boersch phase plate in the back focal plane of the objective lens. The object was then reconstructed from the two complementary images. The Fourier ring correlation (FRC) between the test object and the reconstructed image gives a quantitative measure for the influence of the geometry of the Boersch phase plate. The two-fold geometry of the supporting rods (figure 1a) leads to an unrecoverable loss of information at certain spatial frequencies (centro-symmetric frequencies at rod position are removed). Part of these frequencies can be retrieved from the Friedel pairs in the back focal plane of the objective lens, if a noncentrosymmetrical geometry is used. Figure 1 b shows a possible 3-fold geometry, figure 1c illustrates the resulting improved contrast transfer. The effect is further illustrated in figure 2, which shows the situation for a model crystal (a). In case of a 2-fold symmetry the reconstructed lattice obtains direction dependent features (b), resulting from the loss of structure factors on the vertical axis (b'). For 3-fold symmetry the reconstruction appears isotropic (c) and diffraction spots are also apparent on the vertical axis (c'). Correcting the structure factor amplitudes further improves the reconstruction of the object. Almost all information is recovered (see figure $1 \mathrm{c}$ and $2 \mathrm{~d}$, d') if structure factors, which are in areas shaded by the supporting rods, are multiplied by a factor of two. The described new properties make the Boersch phase plate a promising candidate for high resolution phase contrast TEM. Additionally, the electrostatic phase plate should allow an easier imaging mode switching. Both images, phase contrast and bright field, can be recorded with the phase plate positioned on the optical axis simply by switching the potential on and off. In combination with a spherical aberration corrected objective lens one single phase contrast image will then be enough to obtain the whole frequency spectrum, therefore giving maximal object information. 

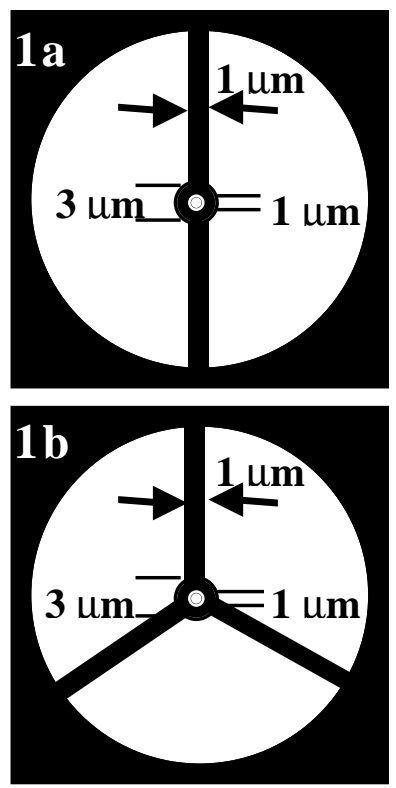

Figure 1. Two possible geometries of the Boersch phase plate: 2-fold symmetry (a) and 3-fold symmetry (b). The phase diameter is on the order of $100 \mu \mathrm{m}$. (c) FRCs between test object and a simulated image reconstruction for the case of phase contrast imaging with 2-fold (dark grey), 3-fold symmetrical (black) Boersch phase plate and for subsequent Friedel pair correction (light grey). The improvement in contrast transfer at low spatial frequencies after Friedel pair correction is obvious.

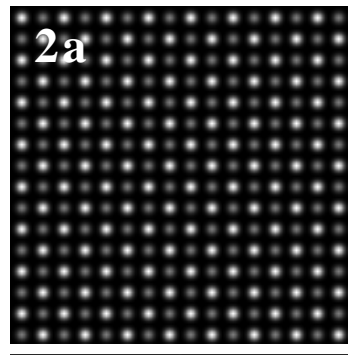

$1 \mathrm{c}$
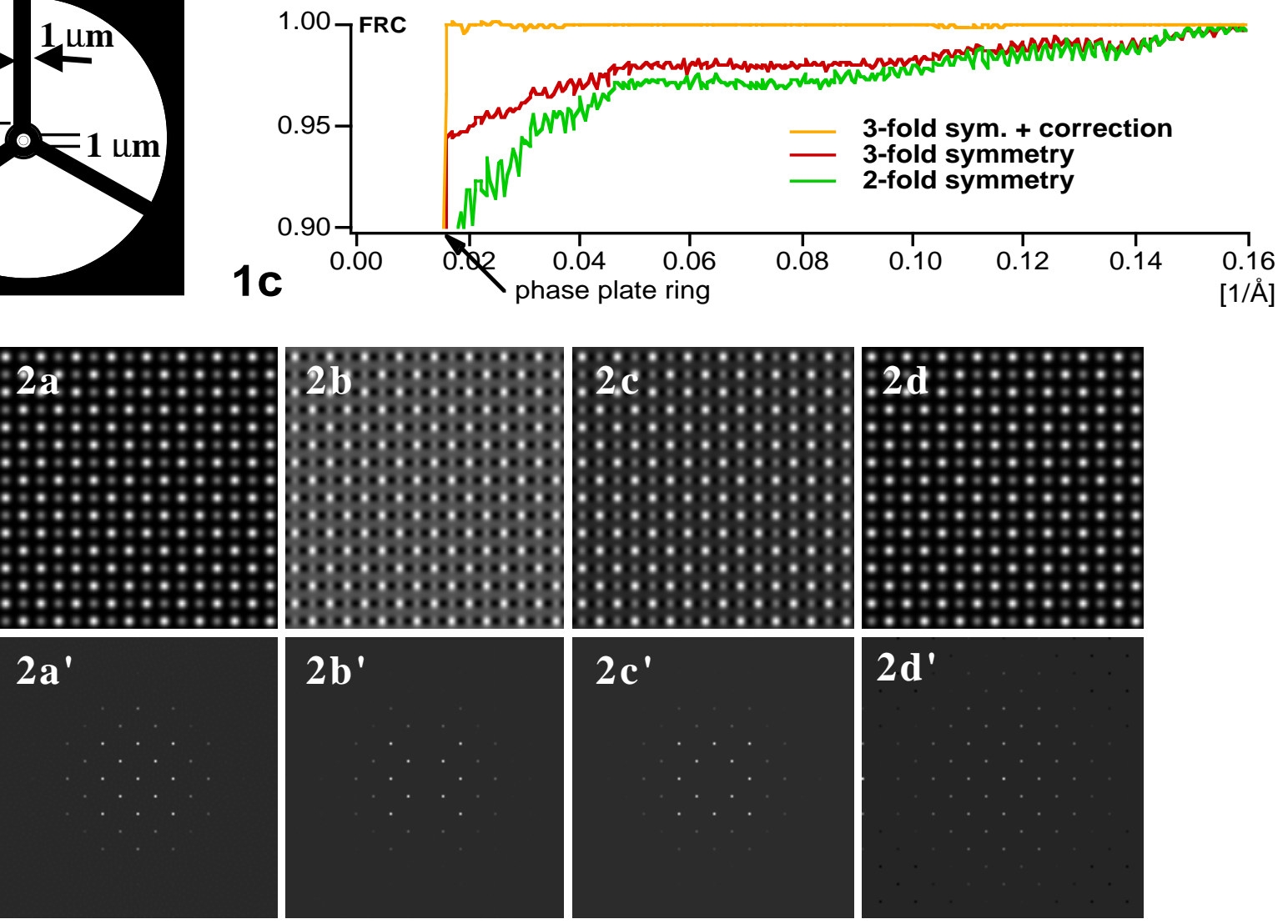

Figure 2. Influence of the Boersch phase plate symmetry on the reconstruction from simulated images of a model-crystal. (a) and (a'): original crystal lattice and its power spectrum. Simulation with a 2-fold symmetry Boersch phase plate leads to direction dependent features (b), resulting from a total loss of information on the vertical axis in the power spectrum ( $\left.b^{\prime}\right)$. This is not the case for 3-fold symmetry, as can be seen in (c) and (c'). After Friedel pair correction apparently all object information is restored ( $\mathrm{d}$ and $\mathrm{d}$ ').

Patent pending for the proposed geometry and structure factor correction.

[1] R. Danev, K. Nagayama, Ultramicroscopy, Vol. 88, No.4, pp. 243-252 (2001)

[2] H. Boersch, Z. Naturforsch. 2a, pp. 615 (1947)

[3] T. Matsumoto, A.Tonomura, Ultramicroscopy, Vol. 63, No.1, pp. 5-10 (1996)

[4] see e.g. abstract at this conference: Majorovits et al. 